\title{
Evaluation Value of Portable Color Doppler Ultrasound for Arteriovenous Fistula Stenosis in Patients with Maintenance Hemodialysis
}

\author{
Sujuan Wu ${ }^{1}$, Lingjun Shen ${ }^{1}$, Wenyu Gong ${ }^{2,}$ * \\ ${ }^{1}$ Department of Dongpu Hemodialysis Center, Dongpu Branch of the First Affiliated Hospital, Jinan University, Guangzhou, China \\ ${ }^{2}$ Division of Nephrology, Department of Medicine, The First Affiliated Hospital, Jinan University, Guangzhou, China
}

Email address:

380069679@qq.com (Wenyu Gong)

${ }^{*}$ Corresponding author

To cite this article:

Sujuan Wu, Lingjun Shen, Wenyu Gong. Evaluation Value of Portable Color Doppler Ultrasound for Arteriovenous Fistula Stenosis in Patients with Maintenance Hemodialysis. American Journal of Internal Medicine. Vol. 8, No. 4, 2020, pp. 143-147.

doi: 10.11648/j.ajim.20200804.11

Received: April 23, 2020; Accepted: May 15, 2020; Published: May 28, 2020

\begin{abstract}
Objective: By comparing the evaluation effect of traditional physical examination (PE) and portable color Doppler ultrasound on arteriovenous fistula (AVF), to explore the value of portable ultrasound in the diagnosis of abnormal fistula lesions in patients with maintenance hemodialysis (HD). Methods: 57 patients with maintenance HD from Dongpu HD center, the first affiliated hospital of Jinan university from September 2019 to March 2020 were selected. The PE was performed by specialist nurses, and AVF was evaluated by portable ultrasound, and the general data of the patients were collected. Results: The average age of the HD patients was 58.98 years, including 31 males (54.39\%). The median of HD duration was 56 months, and 22 patients $(28.60 \%)$ had diabetes mellitus. The prevalence of fistula stenosis was $17.54 \% .50 \%$ PE was abnormal in fistula stenosis group, which was higher than that in non-fistula stenosis group $(10.64 \%, \mathrm{P}=0.012)$. The $\kappa$ index was 0.394 in the consistency assessment between $\mathrm{PE}$ and portable ultrasound for evaluating the fistula stenosis $(\mathrm{P}=0.003)$. The incidence of fistula sound reduction, blood flow less than $200 \mathrm{~mL} / \mathrm{min}$ during treatment and abnormal arm lifting test in patients with fistula stenosis were all higher than those without fistula stenosis (all $\mathrm{P}<0.05$ ). The results of multivariate logistic regression analysis showed that abnormal PE was closely related to fistula stenosis $(\mathrm{OR}=8.400,95 \%$ confidence interval $(\mathrm{CI})=1.786-39.498$, $\mathrm{P}=0.007)$. Conclusion: Portable color Doppler ultrasound could effectively diagnose AVF stenosis in maintenance HD patients, especially when the traditional PE was abnormal, timely portable ultrasound evaluation was conducive to early intervention and extend the life of AVF.
\end{abstract}

Keywords: Hemodialysis, Arteriovenous Fistula, Stenosis, Physical Examination, Ultrasound

\section{Introduction}

Chronic kidney disease (CKD) had become a global disease and a severe public health problem worldwide. Despite the widespread use of interventions to slow the progression of CKD, the burden of end-stage renal disease (ESRD) was still heavy, and China was also facing this challenge $[1,2]$. According to the epidemiological survey in 2012, the prevalence of adult CKD patients in China was as high as $10.8 \%$, and the number was estimated to be 119 million, which resulted in a huge medical burden [3]. Hemodialysis (HD) was one of the important treatments for patients with ESRD, and good vascular access was the basic condition of HD therapy. Arteriovenous fistula (AVF) was the first choice for HD vascular access [4]. Poor function or occlusion of AVF would directly affect the treatment and the occurrence of complications of ESRD. Therefore, the structure and function of AVF should be regularly evaluated to ensure the adequacy of dialysis for patients and reduce complications [5, 6].

Angiography was the gold standard for the detection and evaluation of AVF with high accuracy. However, as an invasive examination, the application of angiography in routine examination and follow-up of uremia patients was 
under restrictions $[7,8]$. In clinic, the specialist nurses in the HD center regularly perform physical examination (PE) of AVF, including appearance, tremor of fistula, sound of AVF blood flow and blood flow up to $200 \mathrm{ml} / \mathrm{min}$ during treatment (that could meet the needs for treatment) or not. However, PE could be affected by subjective factors without clear standard. Worse more, abnormal PE often indicated more serious fistula lesions, thus, the lesions in early stage was easy to miss. As a non-invasive examination, color Doppler ultrasound could immediately evaluate the structure (such as inner diameter, intimal thickness, etc.), function and hemodynamics of AVF, which was conducive to the early detection of AVF structure and functional abnormalities. Therefore, ultrasound examination has been increasingly valued by clinical medical staff, particularly the portable ultrasound was easy to operate and the result was immediate and visual [9-11].

The purpose of this study was to explore the value of portable ultrasound in the diagnosis of abnormal fistula lesions by comparing the evaluation effect between traditional PE and portable color Doppler ultrasound in patients with maintenance $\mathrm{HD}$, so as to provide reference for clinical practice.

\section{Method}

\subsection{Study Population}

A total of 57 patients with HD were collected from the Dongpu HD center, the first affiliated hospital of Jinan university from September 2019 to March 2020, with an average age of 58.98 years, including 31 males and 26 females. Basic diseases include: 28 cases of chronic glomerulonephritis $(49.12 \%), 22$ cases of diabetic nephropathy (DN, 38.60\%), 2 cases of obstructive nephropathy $(3.51 \%), 2$ cases of polycystic kidney disease $(3.51 \%)$ and 3 cases of the others $(5.26 \%$, Figure 1$)$. Inclusion criteria: (1) age $\geq 18$ years; (2) HD duration $\geq 3$ months and weekly treatment time $\geq 12$ hours; (3) HD performed by AVF; (4) informed consent and voluntary participation of the patient or family member. The exclusion criteria were: AVF dilatation or recanalization was operated within 3 months before admission or lack of clinical data.

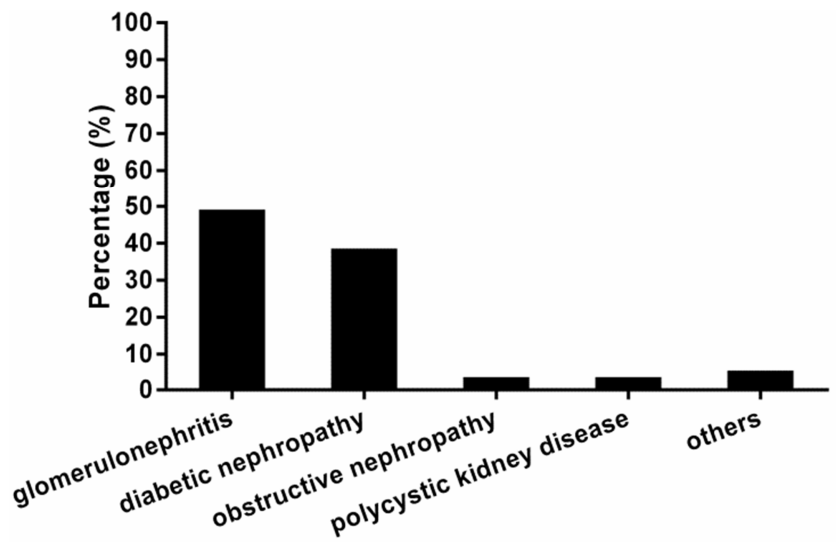

Figure 1. Distributions of basic diseases.

\subsection{Clinical Data Collection}

Demographic data (age, gender, HD duration, etc.) and laboratory results (urea clearance index $\mathrm{K} / \mathrm{tv}$ value, potassium, phosphorus, calcium, albumin, hemoglobin, and intact parathyroid hormone (iPTH)) were collected.

\subsection{Physical Examination}

The patient's fistula was physically examined by trained HD specialist nurses, including skin (intact/damage), tremor (normal/weaker), fistula sound (normal/weaker), blood flow (up to/ less than $200 \mathrm{~mL} / \mathrm{min}$ during treatment) and arm lifting test (normal/abnormal). If there was any abnormality of fistula tremor, sound, blood flow during treatment and arm lifting test, it would be considered as abnormal PE.

\subsection{Portable Color Doppler Ultrasound Evaluation}

SonoSite hand-carried ultrasound (SonoSite Inc., Bothell, WA, USA) was used to evaluate the structure and function of AVF. When the internal diameter of the anastomosis was less than $2.5 \mathrm{~mm}$, or the internal diameter of the venous was less than $3.0 \mathrm{~mm}$ and reduced $50 \%$ compared with the adjacent normal vessels, it was considered as the fistula stenosis [12].

\subsection{Statistical Analysis}

Continuous variables were represented as mean \pm standard deviation (SD), while non-parametric variables were represented as median and quartile ranges. Categorical variables were expressed as frequency and percentage. Student's t-tests or nonparametric tests were used to compare continuous variables between groups. Differences between categorical variables were analyzed by chi-square tests or double-tailed Fisher exact tests, as applicable.

The $\kappa$ index was used to assess the consistency between $\mathrm{PE}$ and color Doppler ultrasound for evaluating the stenosis of AVF. The influencing factors of fistula stenosis were analyzed by multivariate logistic regression analysis.

All values were two-tailed, and $P<0.05$ was considered statistically significant. Data were analyzed using IBM SPSS Statistics version 25.0 for Windows (IBM, Armonk, NY, USA).

\section{Result}

\subsection{Clinical Characteristics}

The average age of the HD patients was 58.98 years, including 31 males $(54.39 \%)$. The median of HD duration was 56 months, and 22 patients $(28.60 \%)$ had diabetes mellitus. $29.82 \%$ patients had smoking and $28.07 \%$ of them drank alcohol. The prevalence of fistula stenosis was $17.54 \%$. There were no statistically significant differences in age, gender, prevalence of diabetes mellitus, smoking, drinking, $\mathrm{K} /$ tv value, potassium, phosphorus, calcium, albumin, hemoglobin and iPTH between patients in the fistula stenosis group and those without fistula stenosis (all $\mathrm{P}>0.05$, Table 1). 
Table 1. Differences of demographic and clinical characteristics.

\begin{tabular}{lllll}
\hline & Fistula non-stenosis (N=47) & Fistula stenosis (N=10) & Total (N=57) & P \\
\hline Age (years) & $57.53 \pm 15.74$ & $65.80 \pm 10.59$ & $58.98 \pm 15.22$ & 0.120 \\
Gender (male: female) & $25: 22$ & $6: 4$ & $31: 26$ & 0.966 \\
HD duration (months) & $60(29-77)$ & $36(23-66)$ & $56(28-75)$ & 0.356 \\
DM & $16(34.04 \%)$ & $6(60 \%)$ & $22(38.60 \%)$ & 0.241 \\
Smoking (N/\%) & $13(27.66 \%)$ & $4(40 \%)$ & $17(29.82 \%)$ & 0.694 \\
Drinking (N/\%) & $12(25.53 \%)$ & $4(40 \%)$ & $16(28.07 \%)$ & 0.591 \\
K/tv value & $1.43 \pm 0.30$ & $1.42 \pm 0.14$ & $1.43 \pm 0.28$ & 0.889 \\
Potassium (mmol/L) & $5.06 \pm 0.69$ & $5.07 \pm 0.67$ & $5.06 \pm 0.68$ & 0.976 \\
Phosphorus (mmol/L) & $2.44 \pm 0.81$ & $2.32 \pm 0.60$ & $2.42 \pm 0.77$ & 0.676 \\
Calcium (mmol/L) & $2.24 \pm 0.21$ & $2.32 \pm 0.40$ & $2.26 \pm 0.25$ & 0.537 \\
Albumin (mmol/L) & $38.90 \pm 2.52$ & $38.50 \pm 3.14$ & $38.83 \pm 2.61$ & 0.664 \\
Hemoglobin & $104.22 \pm 14.55$ & $111.30 \pm 16.10$ & $105.46 \pm 14.93$ & 0.175 \\
iPTH & $411(237-626)$ & $402(185-598)$ & $411(236-600)$ & 0.941 \\
\hline
\end{tabular}

DM: diabetes mellitus; HD: hemodialysis; iPTH: intact parathyroid hormone.

$\mathrm{P}$ value for analysis of comparison between patients without fistula stenosis and patients with fistula stenosis.

\subsection{AVF Assessment}

According to the results of PE, 2 patients (3.51\%) had broken skin in the AVF. There were 4 fistula tremor (7.02\%) and 2 fistula sound $(3.51 \%)$ reduced. There were 5 blood flow $(8.77 \%)$ less than $200 \mathrm{~mL} / \mathrm{min}$ during treatment and 5 patients $(8.77 \%$ ) had abnormal arm lifting test (Figure 2). $50 \% \mathrm{PE}$ was abnormal in fistula stenosis group, which was higher than that in non-fistula stenosis group $(10.64 \%$, $\mathrm{P}=0.012$ ). The $\kappa$ index was 0.394 in the consistency assessment between $\mathrm{PE}$ and portable ultrasound for evaluating the fistula stenosis $((\mathrm{P}=0.003$, Table 2 and Figure $3)$.

In the fistula stenosis group, 1 patient had broken skin in the AVF. There were 2 fistula tremor and 2 fistula sound reduced. There were 3 blood flow less than $200 \mathrm{~mL} / \mathrm{min}$ during treatment and 4 patients $(8.77 \%)$ had abnormal arm lifting test. Compared with patients without fistula stenosis, the incidence of fistula sound reduction, blood flow less than $200 \mathrm{~mL} / \mathrm{min}$ and abnormal incidence of arm lifting test were all higher in patients with fistula stenosis (all $\mathrm{P}<0.05$, Figure 3 ).

Table 2. The consistency assessment between PE and portable ultrasound for evaluating the fistula stenosis.

\begin{tabular}{|c|c|c|c|c|}
\hline & & \multicolumn{2}{|c|}{ Portable ultrasound evaluate } & \multirow{2}{*}{ Total } \\
\hline & & fistula non-stenosis & fistula stenosis & \\
\hline \multirow{2}{*}{ PE } & normal & 42 & 5 & 47 \\
\hline & abnormal & 5 & 5 & 10 \\
\hline Total & & 47 & 10 & 57 \\
\hline
\end{tabular}

PE: physical examination.

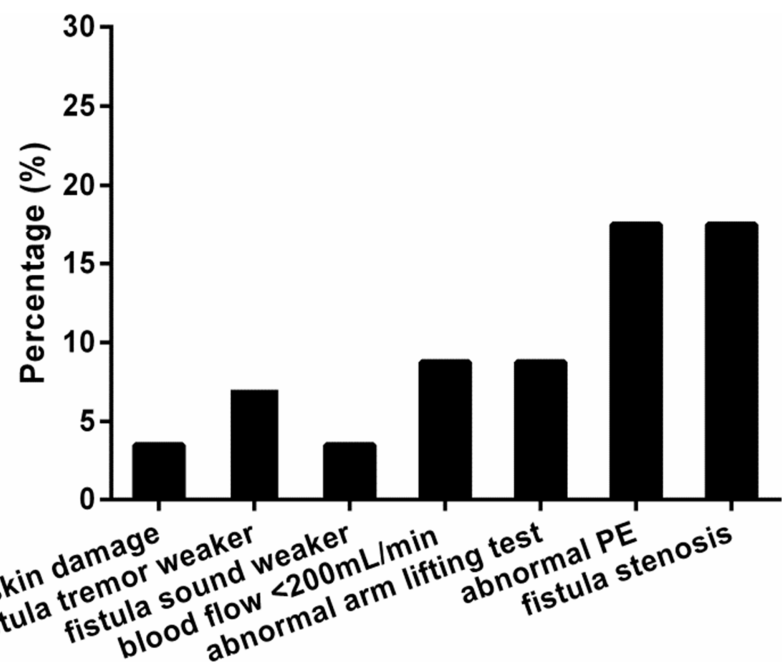

Figure 2. Percentage of PE abnormality and fistula stenosis in HD patients.

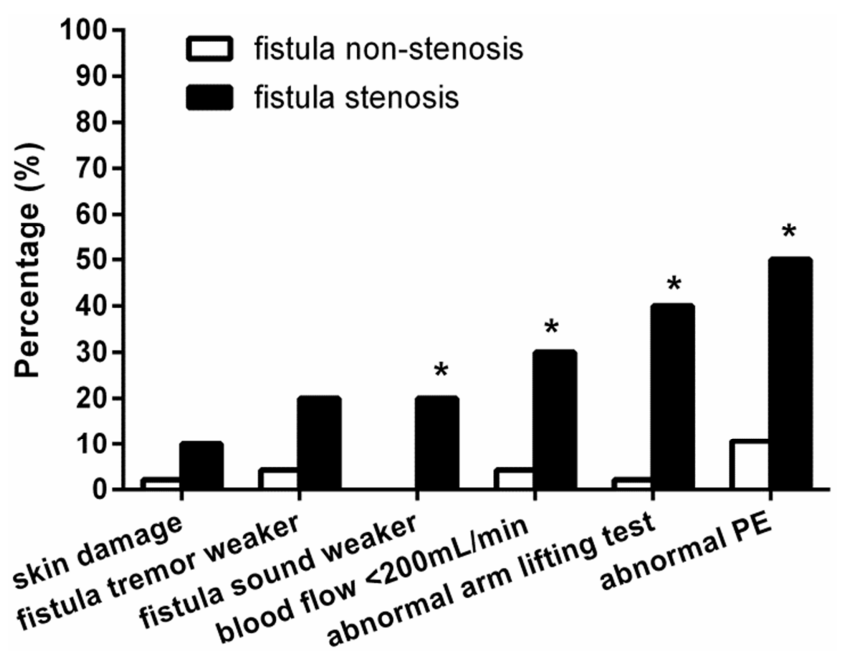

(*indicated comparison with fistula non-stenosis group, $\mathrm{p}<0.05$ ).

Figure 3. Percentage of PE abnormality between patients without fistula stenosis and patients with fistula stenosis. 


\subsection{Influencing Factors of Fistula Stenosis}

To investigate the independent influencing factors of fistula stenosis, multivariate logistic regression analysis was performed with demographic data, laboratory examination results and PE indicators as independent variables. The results showed that abnormal $\mathrm{PE}$ was closely related to fistula stenosis $(\mathrm{OR}=8.400,95 \%$ confidence interval $(\mathrm{CI})=1.786-39.498, \mathrm{P}=0.007$, Table 3$)$.

Table 3. Multivariate logistic regression analysis for the fistula stenosis ( $0=$ fistula non-stenosis, $1=$ fistula stenosis).

\begin{tabular}{lllll}
\hline & \multirow{2}{*}{ OR } & \multicolumn{2}{l}{$\mathbf{9 5 \%}$ CI for OR } & \multirow{2}{*}{ P } \\
\cline { 3 - 4 } & & Lower & Upper & \\
\hline Abnormal PE & 8.400 & 1.786 & 39.498 & 0.007 \\
\hline
\end{tabular}

CI: confidence interval; OR: odds ratio

Variables include age, gender $(1=$ male, $2=$ female), diabetes mellitus, hemoglobin and abnormal PE ( $0=$ normal, $1=$ abnormal $)$.

\section{Discussion}

AVF was known as the lifeline of HD patients, so a fast and effective non-invasive detection method was needed in clinic to evaluate the structure and function of AVF. This study showed that the prevalence of fistula stenosis was $17.54 \%$. 50\% PE was abnormal in fistula stenosis group, which was higher than that in non-fistula stenosis group (10.64\%, $\mathrm{P}=0.012$ ). The $\kappa$ index was 0.394 in the consistency assessment between $\mathrm{PE}$ and portable ultrasound for evaluating the fistula stenosis $(\mathrm{P}=0.003)$. The incidence of fistula sound weaker, blood flow (less than $200 \mathrm{~mL} / \mathrm{min}$ during treatment) and abnormal arm lifting test in patients with fistula stenosis were all higher than those without fistula stenosis (all $\mathrm{P}<0.05$ ). The results of multivariate logistic regression analysis showed that abnormal PE was closely related to fistula stenosis $(\mathrm{OR}=8.400,95 \% \mathrm{CI}=1.786-39.498$, $\mathrm{P}=0.007$ ).

$\mathrm{HD}$ as one of the main treatment methods of uremia, good vascular access was its important basis. K/DOQI guidelines clearly suggested that AVF was recommended for HD in patients with ESRD, which was conducive to reducing complications, improving the quality of life and improving the prognosis of patients [13]. The patency and good functional status of AVF was very important for HD. Regular evaluation could find out the complications of AVF in the early stage, so as to early clinical intervention or treatment [14]. Angiography was the gold standard for the evaluation of AVF, but its clinical application was restricted by high technical requirements, expensive, exposure to radiation, need for contrast agent and invasive factors. Color Doppler ultrasound was gradually favored by clinicians because of its advantages of non-invasive, safe, cheap, simple technology and visual result. It could timely conduct quantitative observation on vascular diameter, flow rate and filling status, which was helpful for clinical detection and analysis [15].

PE for AVF was one of the routine works of HD specialist nurses. Qualitative observation of the appearance, diameter, vascular direction and blood flow velocity of AVF through visual examination, palpation and auscultation was helpful to find out the malfunction of AVF. However, the PE was subjective without clear quantitative standard, and when the PE showed abnormal, it usually indicated a serious lesion. In our study, the consistency between PE abnormalities and fistula stenosis diagnosed by portable ultrasound was low, which indicated that PE could found out the fistula stenosis in early stage in time. The multivariable logistic analysis showed that the risk of fistula stenosis increased by 8.4 times when PE showed abnormal, which suggested that portable ultrasound detection should be carried out immediately when PE was abnormal, so as to detect the problem as early as possible and extend the life of AVF. Previous studies had found that age, hypotension and diabetes mellitus were independent risk factors for fistula dysfunction, so the examination frequency of these patients should be strengthened $[16,17]$. Similar results didn't found in this study, which might because of the small sample size, thus the sample size should be increased in the further studies.

This study had some advantages and limitations. First of all, we evaluated the value of portable ultrasound in the diagnosis of poor function of AVF. Secondly, ultrasound, as a convenient, non-invasive and visual tool, had a high predictive value for AVF stenosis and was suitable for clinical application. Limitations included the following points: the sample size was relatively small; due to only one center was included, multicenter data and follow-up studies were necessary; and lack of some demographic characteristics and biochemical indexes involved in AVF stenosis.

\section{Conclusion}

Since the life expectancy of patients with ESRD was gradually extended, the problems caused by poor fistula function were widely concerned. Portable color Doppler ultrasound could timely diagnose fistula stenosis, which was worth promoting in clinical work. Furthermore, portable ultrasound examination should be carried out according to the age, complications and PE results of HD patients, so as to effectively prevent and treat the dysfunction of AVF and improve the survival rate and quality of life of patients.

\section{Competing Interests}

The authors declare that they have no competing interests.

\section{Acknowledgements}

The authors would like to acknowledge all patients and their families for participating in this study.

\section{References}

[1] Coresh J, Selvin E, Stevens LA, et al. Prevalence of chronic kidney disease in the United States [J]. JAMA, 2007, 298 (17): 2038-2047. 
[2] Stenvinkel P. Chronic kidney disease: a public health priority and harbinger of premature cardiovascular disease [J]. J INTERN MED, 2010, 268 (5): 456-467.

[3] Zhang L, Wang F, Wang L, et al. Prevalence of chronic kidney disease in China: a cross-sectional survey [J]. LANCET, 2012, 379 (9818): 815-822.

[4] Gökhan I, Ertap E, Sahin B, et al. The Clinical Utility of Vascular Mapping With Doppler Ultrasound Prior to Arteriovenous Fistula Construction for Hemodialysis Access [J]. J Vasc Access, 2013, 14 (1), 83-88.

[5] Timmy L, Joyce Q, Mae T, et al. Tradeoffs in Vascular Access Selection in Elderly Patients Initiating Hemodialysis with a Catheter. Am J Kidney Dis. 2018, 72 (4): 509-518.

[6] McGrogan DG, Maxwell AP, Khawaja AZ, et al. Current tools for prediction of arteriovenous fistula outcomes. Clin Kidney $\mathrm{J}$ 2015; 8 (3): 282-289.

[7] Yevzlin AS, Chan MR, Asif A. Hand Ischemia in a Patient With an Arteriovenous Fistula [J]. Am J Kidney Dis, 2016, 67 (3): 512-515.

[8] Jalandhara N, Balamuthusamy S, Skaria S, et al. Persistent Median Artery As A Cause Of Nonmaturing AVFistula [J]. Semin Dial, 2015, 28 (5): 552-557.

[9] Jamil M, Usman R. Predictive parameters for successful functional maturation of native arteriovenous fistula $[\mathrm{J}]$. J Ayub Med Coll Abbottabad, 2015, 27 (4): 821-824.

[10] Masengu A, McDaid J, Maxwell AP, et al. Preoperative radial artery volume flow is predictive ofarteriovenous fistula outcomes [J]. J Vasc Surg, 2016, 63 (2): 429-435.

[11] Renaud CJ, Leong CR, Bin HW, et al. Effect of brachial plexus block-driven vascular access planning on primary distal arteriovenous fistula recruitment and outcomes [J]. J Vasc Surg, 2015, 62 (5): 1266-1272.

[12] Jones RG, Inston NG, Brown T. Arteriovenous fistula salvage utilizing a hybrid vascular graft $[\mathrm{J}]$. J Vasc Access, 2014, 15 (2): $135-137$.

[13] Moore LW, Byham-Gray LD, Scott Parrott J, et al. The mean protein intake at different stages of chronic kidney disease is higher than current guidelines [J]. Kidney Int, 2013, 83 (4): 724-732.

[14] Tanner NC, da Silva AF. Medical Adjuvant Treatment to Improve the Patency of Arteriovenous Fistulae and Grafts: A Systematic Review and Meta-analysis [J]. Eur J Vasc Endovasc Surg, 2016, 52 (2): 243-252.

[15] Lomonte C, Meola M, Petrucci I, et al. The key role of color Doppler ultrasound in the work-up of hemodialysis vascular access [J]. Semin Dial, 2015, 28 (2): 211-215.

[16] Miller PE, Tolwani A, Luscy CP, et al. Predictors of adequacy of arteriovenous fistulas in hemodialysis patients. Kidney Int. 1999 Jul; 56 (1): 275-280.

[17] Charmaine E Lok, Michael Allon, Louise Moist, et al. Risk equation determine unsuccessful communication events and failure to maturation in arteriovenous fistulas (REDUCE FTM I). J Am Soc Nephrol, 2006, 17 (11): 3204-3212. 